\title{
Mechanical sensors
}

\section{Hydraulic Turbine Vortex Detection and Visualization Using Strain Gauge Sensor}

\author{
Irina Murgan ${ }^{1}$, Gabriel Vasile $^{1 * *}$, Cornel loana ${ }^{1 *}$, Stéphane Barre $^{2}$, and Thierry Lora-Ronco ${ }^{3}$ \\ ${ }^{1}$ GIPSA-lab, Université Grenoble Alpes, Grenoble F-38400, France \\ ${ }^{2}$ LEGI, UMR 5519, Grenoble F-38041, France \\ ${ }^{3}$ EDF-DTG, Grenoble F-38040, France \\ *Member, IEEE \\ ** Senior Member, IEEE
}

Manuscript received June 21, 2017; revised July 23, 2017 and August 10, 2017; accepted August 22, 2017. Date of publication September 8, 2017; date of current version September 26, 2017.

\begin{abstract}
Hydraulic machinery monitoring using nonintrusive sensors is today's trend in the hydroelectric power industry. The phenomena of cavitation vortex occurs mostly at off-design regimes, which reduces the turbine's efficiency. In this article, we propose a mathematical model for vortex detection and a 3-D vortex presence visualization. Its visualization is constructed on the gyroscope principle, translating the strain gauge vertical elongations into a nutation movement. The mathematical model for vortex detection computes the data statistical moments. The model is tested on experimental data recorded using an extensometer.
\end{abstract}

Index Terms—Mechanical sensors, extensometer, hydraulic turbine monitoring, pdf estimation, vortex detection.

\section{INTRODUCTION}

Rotating machines, such as rotary engines, turbines, or wheels, are often exposed during their operational lifetime to several undesired phenomenon: vibrations, mechanical stress or cavitation [1]. Such phenomena can lead to premature wear of the machines or worse, to their destruction [2]. It is particularly the case for the hydraulic turbines, because of their dimensions, diversity and components complexity.

A hydraulic turbine is mainly composed by a specific number of guide vanes that control the water flow rate, the moving part (rotorblade assembly), the draft tube controlling the water evacuation and the electrical generator that transform the mechanical energy into electrical energy. The turbine is designed to transform the water kinetic energy with maximum of efficiency, under certain operating conditions, called best efficiency operating point.

Nowadays, the expansion of renewable energy sources (solar, wind, etc.) may affect, by their intermittent nature, the stability of the power system. In order to maintain the balance between energy production and consumption, the hydroelectric power plants are often used. Thus, in order to be able to respond to a various demands of the power grid, the hydraulic turbines tend to have their operation range extended (i.e., operate under off-design conditions). When a hydraulic turbine operates under off-design conditions, the phenomena of vortex cavitation is likely to appear [3]. The conditions for vortex cavitation appearance are an unsteady flow with water pressure drop under the water vaporization pressure. In this case, the turbine efficiency is decreased as flow instabilities induce mechanical vibrations and large pressure fluctuations. They generate axial and radial efforts together

Corresponding author: Irina Murgan (e-mail: irina.murgan@gipsa-lab.grenobleinp.fr).

Associate Editor: K. Foster.

Digital Object Identifier 10.1109/LSENS.2017.2750402 with the mechanical stress created by the turbine's rotation. Apart from the machine mechanical damage, output power fluctuations are generated, putting at risk the electrical grid stability. Therefore, it is important to know at every instance of time, the amplitude of these instabilities and to be able to control them. Up to a certain level, the efforts are absorbed by a coupling system composed by bearings, thus avoiding a direct transfer to the electrical generator. In today practice, for hydraulic system monitoring, intrusive pressure sensors are used in order to detect pressure fluctuations. Mathematical simulations and reduce scale tests are also performed for flow prediction and vortex identification [4]-[9]. Due to flow instabilities and turbulences, these methods cannot be generalized, despite the fact that they may provide satisfying results [10]-[12].

Multiple sensors of different types are used in order to perform constant hydropower plant monitoring, especially in the case of the rotational components. In particular, the time evolution of the axial efforts is measured using extensometers. Having the extensometer elongations measurements at the bearing unit level, we propose a novel processing method, in order to visualize the turbine off-design behavior in terms of the generated axial efforts. Our study is based on a mathematical model presented in Section II of this paper. In Section III, the model is applied to a real calibration dataset, for different turbine's operating conditions. Conclusions and perspectives are presented in Section IV.

\section{THEORETICAL FRAMEWORK}

In order to characterize the vortex cavitation presence, we create a mathematical and statistical interpretation of the generated axial efforts. The proposed method performs a conversion of the measured elongations in a way that the axial efforts can be directly interpreted with the nutation movement of a gyroscope (see Fig. 1). This axial effort's measurement is crucial in quantifying the instabilities levels 


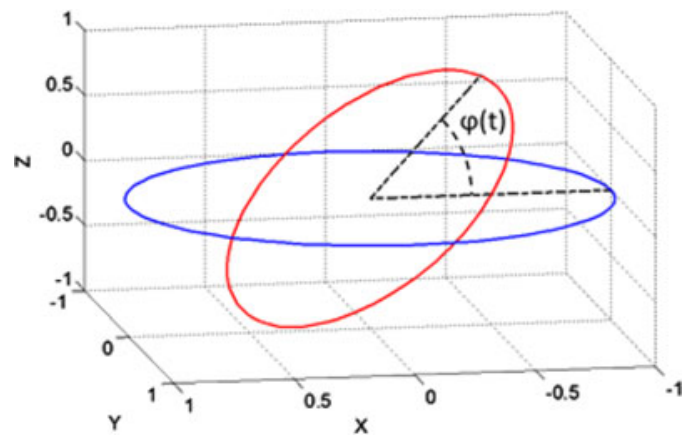

Fig. 1. Three-dimensional rotation of a circle with angle $\varphi$, around $y$-direction.

at the current operational point of the turbine. Similar representations are performed in [13], as part of shaft dynamical analysis. The analogy between the extensometer measurements and the nutation movement provides a new virtual monitoring instrument of the machine instabilities. In the proposed framework, we expect to obtain a minimum level of instabilities for the best efficiency point and a larger one for lower efficiency operating points.

\section{A. Mathematical Model}

Let consider a circle $C$ in a spherical coordinates system $(r, \varphi, \theta)$, to which we apply a set of three rotation matrixes $R_{x}, R_{y}$ and $R_{z}$ for each of the three directions $\mathrm{x}, \mathrm{y}$, and respectively, $\mathrm{z}$. The rotated circle $C_{r}$ is given by the following system of equations:

$$
C_{r}=R_{z} \times R_{x} \times R_{y} \times C,
$$

Here, $t \in[0, T]$, with $T$ being the duration of one full rotation of a disk around the $O z$ axis and $\varphi, \theta \in[0,2 \pi]$. We define the rotation angle $\varphi$ as a function of the extensometer elongations measurements $\mathrm{S}_{\text {in }}(t)$ :

$$
\varphi(t)=\operatorname{arctg}\left(\frac{\mathrm{S}_{i n}(t)}{r}\right)
$$

Let us consider a nonlinear operator: $X$. If the $X$ operator is applied to our signal $S_{i n}(t)$, the response is given by

$$
X\left\{S_{i n}(t)\right\}=\frac{1}{1+S_{i n}^{2}(t)}
$$

We rewrite the equations system (1) as follows:

$$
\begin{aligned}
C_{r}=r & \cdot\left(\begin{array}{ccc}
\sin [\theta(t)] & \cos [\theta(t)] & -\sin [\theta(t)] \\
\cos [\theta(t)] & \sin [\theta(t)] & \cos [\theta(t)] \\
0 & -\sin [2 \varphi(t)] & \sin [\varphi(t)]
\end{array}\right) \\
& \cdot\left(\begin{array}{ccc}
\cos [\alpha(t)] & 0 & -\cos [\alpha(t)] \\
0 & \cos [\alpha(t)] & 0 \\
0 & \sin [\alpha(t)] & 0
\end{array}\right) \cdot\left(\begin{array}{c}
\cos ^{2}[\varphi(t)] \\
\cos [\varphi(t)] \\
1
\end{array}\right) \\
= & \cdot M(t) \cdot\left(\begin{array}{c}
\cos ^{2}[\varphi(t)] \\
\cos [\varphi(t)] \\
1
\end{array}\right) \stackrel{r=1}{\rightarrow} M(t) \cdot\left(\begin{array}{c}
X\left\{S_{\text {in }}(t)\right\} \\
\left(X\left\{S_{\text {in }}(t)\right\}\right)^{\frac{1}{2}} \\
1
\end{array}\right) .
\end{aligned}
$$

In (4), the unity circle is considered. Further, we will study the response of different types of input signals, when we will apply the proposed operator $\mathbf{X}$. The input signal pattern is a steady state components $(t)$ (which corresponds to a deterministic process, in this

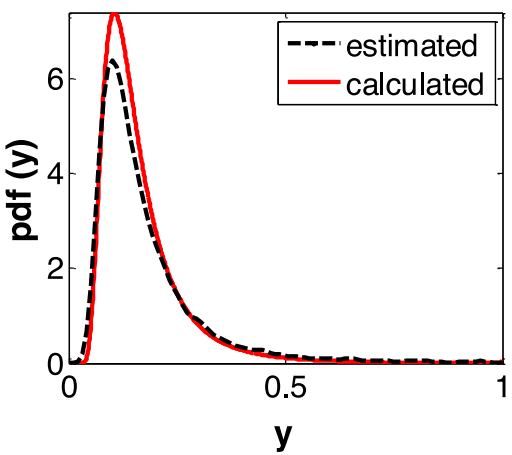

Fig. 2. Probability density function of the $X$ transform of a Gaussian noise $\sim \mathrm{N}(2.5,0.5)$ has the mean $=0.15$ and the variance $=0.01$ if it is computed using (8), and it has the mean $=0.17$ and the variance $=0.3$ if it is numerical estimated.

case the turbine rotation frequency) and the additive noise

$$
S_{i n}(t)=s(t)+n(t) .
$$

We have chosen to express in this way the signal pattern, because we will perform a statistical analysis later. Generally, the probability density function for an experimental data can be predicted, if it has a Gaussian or sinusoidal shape [14]. Deterministic signals have periodic amplitudes that allow harmonically decomposition related to sin waves; thus, $s(t)$ will be the sin wave corresponding to the turbine rotation. The additive noise $n(t)$ present in the frequency bandwidth which corresponds to the cavitation vortex, is assumed to be Gaussian.

In the following sections we will analytically estimate the response of each component, i.e., sinusoid and noise, to the $\mathrm{X}$ operator.

\section{B. Gaussian Noise Input}

Let us consider the case of independent and identically distributed Gaussian noise input $n(t)$. The corresponding probability density function, pdf, is given by the following relation:

$$
p_{n}(x)=\frac{1}{\sigma \sqrt{2 \pi}} e^{-\frac{(x-\mu)^{2}}{2 \sigma^{2}}},-\infty<x<\infty .
$$

We want to see how $p_{n}(x)$ is changed if we apply the $\mathrm{X}$ operator. We will perform a probability density function variable change [11]. Let's consider a random and continuous variable $x$, normally distributed, with the probability density function given by (7), shown below. The variable change that we propose is: $x \rightarrow \frac{1}{1+x^{2}}$. Hence, consider the function $X_{n}(x)=\frac{1}{1+x^{2}}$ which is strictly increasing on the interval $(-\infty, 0]$ and strictly decreasing on the interval $[0, \infty)$. On both intervals, $X_{n}$ is continuous and differentiable.

The equation $X_{n}(x)=y$ has the following two solutions: $x_{1}=$ $\sqrt{\frac{1-y}{y}}$ and $x_{2}=-\sqrt{\frac{1-y}{y}}$. Thus, we have

$$
\begin{aligned}
p_{n}(y) & =\frac{p_{n}\left(x_{1}\right)}{\left|X_{n}^{\prime}\left(x_{1}\right)\right|}+\frac{p_{n}\left(x_{2}\right)}{\left|X_{n}^{\prime}\left(x_{2}\right)\right|} \\
& =\frac{1}{\sigma y \sqrt{2 \pi y(1-y)}} \cdot\left[e^{-\frac{\left(\sqrt{\frac{1-y}{y}}-\mu\right)^{2}}{2 \sigma^{2}}}+e^{-\frac{\left(-\sqrt{\frac{1-y}{y}}-\mu\right)^{2}}{2 \sigma^{2}}}\right]
\end{aligned}
$$

for $y \in(0,1)$. An example of the estimated and calculated $X$ transform of a Gaussian noise pdf is displayed in Fig. 2. According to (8), shown below, the pdf domain of definition is restrained to the interval $(0,1)$. 


\section{Sinusoidal With Additive Noise Input}

In this section, we will perform the pdf analysis of the $X$ transform of a sinusoidal wave with Gaussian noise. Consider a sinusoidal wave of the form: $s(t)=A \cdot \sin (2 \pi f t+\theta)$. First, we compute the joint pdf of one sinusoid and an additive noise, supposed to be Gaussian noise. It is given by the convolution of their corresponding pdfs [14]:

$$
\begin{aligned}
p_{S_{i n}}(x) & =\int_{-\infty}^{\infty} p_{s}(x-\tau)^{*} p_{n}(\tau) d \tau \\
& =\frac{1}{\sigma \pi \sqrt{2 \pi}} \int_{0}^{\pi} e^{-\left(\frac{x-A \cos (\theta)}{4 \sigma}\right)^{2}} d \theta
\end{aligned}
$$

where $\sigma$ is the noise's standard deviation; $A$ and $\theta$ are the sinusoidal wave's amplitude and phase. If we develop, in Taylor- MacLaurin series, the exponential term from (9), then $p_{S_{i n}}(x)$ can be rewritten as follows:

$$
p_{S_{i n}}(x)=\frac{\alpha}{\sigma \sqrt{2 \pi}} e^{-\left(\frac{x}{4 \sigma}\right)^{2}}
$$

where $\alpha=\alpha(A, \sigma, N)$ is a constant depending on the sinusoid amplitude, noise standard deviation and the number of Taylor-MacLaurin series terms, $N$. For calculus simplicity, we suppose $N$ is an odd number.

Second, we perform for $p_{S_{i n}}(x)$ the same variable change as in the previous section, and we will get the pdf of $X\left(S_{i n}\right)$ :

$$
p_{X\left(S_{i n}\right)}(y)=\frac{\alpha}{\sigma \sqrt{2 \pi y^{3}(1-y)}} \cdot e^{\frac{y-1}{(4 \sigma)^{2} y}}
$$

The $\mathrm{k}$-th order moments of $p_{X\left(S_{i n}\right)}(y), k \geq 1$ are given by the following relation [15]:

$$
m_{k}=\int_{-\infty}^{\infty} y^{k} \cdot p_{X\left(S_{i n}\right)}(y) .
$$

As mentioned in previous section, $y \in(0,1)$. We perform the variable change $\frac{1-y}{y}=t$, and we obtain

$$
m_{k}=\frac{\alpha}{\sigma \sqrt{2 \pi}} \int_{0}^{\infty} \frac{1}{\sqrt{t}} \cdot \frac{1}{(1+t)^{k}} \cdot e^{-a t} d t
$$

with $a=\frac{1}{(4 \sigma)^{2}}$. If we compute the integral from (13), shown below, for the first moment [16], we get

$$
m_{1}=\pi \cdot \frac{\alpha}{\sigma \sqrt{2 \pi}} \cdot e^{a} \cdot \operatorname{erfc}(\sqrt{a})
$$

As (13) proves, the pdf's moments are functions of sinusoid amplitude and noise standard deviation. The sinusoid describes a deterministic process, hence $A$ is known, and $\sigma$ can be estimated.

In order to test the robustness of our proposed transform, we perform an SNR level test. The moments of first and second order are calculated for a SNR range between -30 and $10 \mathrm{~dB}$. Their variation is displayed in Fig. 3.

\section{EXPERIMENTAL TESTS AND RESULTS}

\section{A. Data Acquisition}

In our experiment, SCAIME Epsimetal extensometer sensor with a resolution better than $1 \mu \mathrm{m} / \mathrm{m}$ [17] is placed on the alternator bearing conical support at $150 \mathrm{~mm}$ distance from its outer edge and at $300 \mathrm{~mm}$ distance from the alternator bearing of a Francis turbine (see Fig. 4).
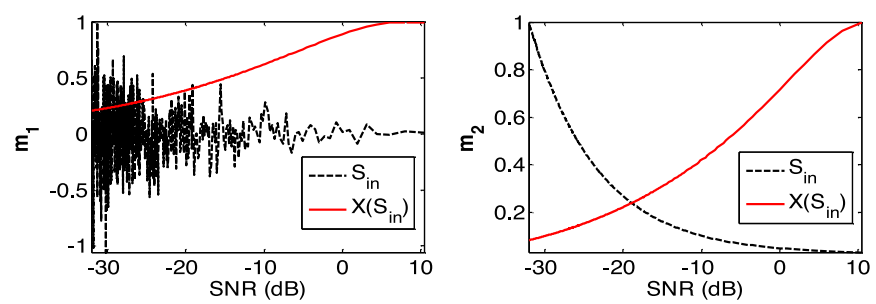

Fig. 3. SNR level test.

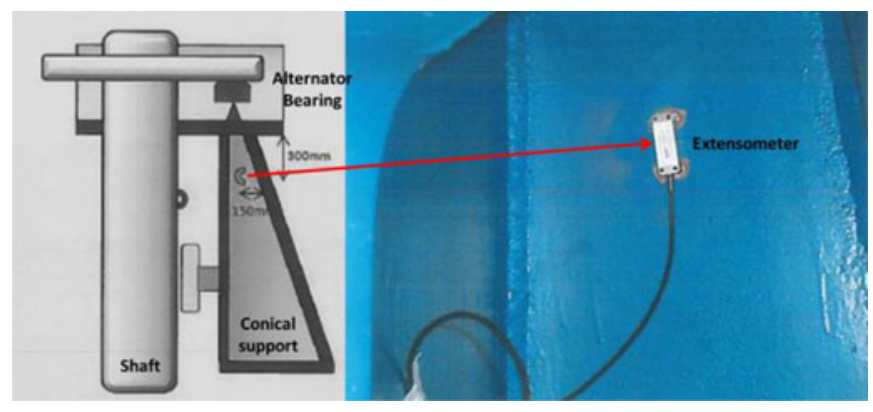

Fig. 4. Extensometer placement on the alternator bearing.

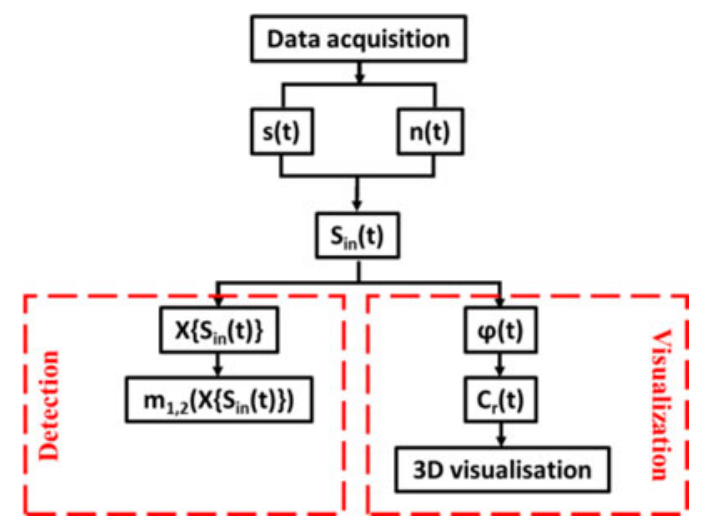

Fig. 5. Measured data processing method.

Using 18 different operating conditions (different water flow rates, between 80 and $350 \mathrm{l} / \mathrm{s}$ ), elongations variations were recorded with a sampling rate of $5120 \mathrm{~Hz}$. The extensometer measurements are related to the turbine mechanical behavior. The link with the hydraulic phenomenon is made by the presence of low frequencies under $1 \mathrm{~Hz}$, in the spectral analysis of the recorded data.

\section{B. Results}

The theoretical framework presented in Part II, will be used now to detect and visualize the cavitation vortex presence (see Fig. 5). The input signal $S_{i n}(t)$ is composed by the sum of one sinusoids, corresponding to the turbine rotation frequency and the noise in the corresponding vortex frequency bandwidth between 0.5 and $1 \mathrm{~Hz}$.

The vortex detection is performed by the computing the first and second order statistical moments of $\mathrm{S}_{i n}$ and $\mathrm{X}\left\{\mathrm{S}_{i n}\right\}$ for several working regimes. In order to apply (13), the sinusoid parameters are constants, regardless the turbine's working regime, and $\sigma$ is the reference noise standard deviation (the $\sigma$ of recorded noise for the optimal working conditions of the turbine). Fig. 6 shows moments variation of $S_{i n}$ and $\mathrm{X}\left\{\mathrm{S}_{i n}\right\}$, for all flow rates. The effect of using the proposed transform 

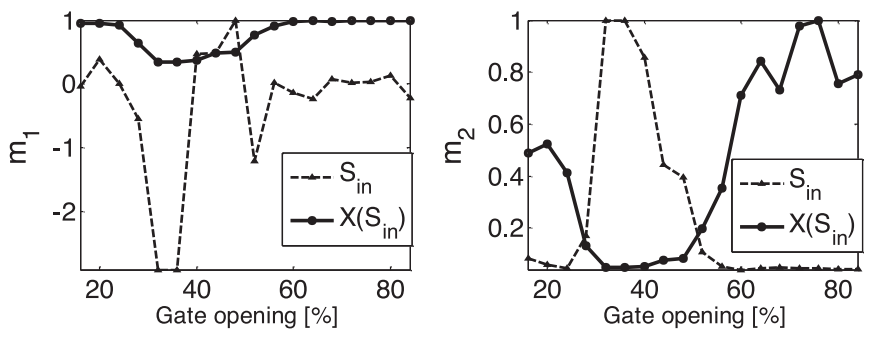

Fig. 6. Statistical moments variation as a function of the gate opening.

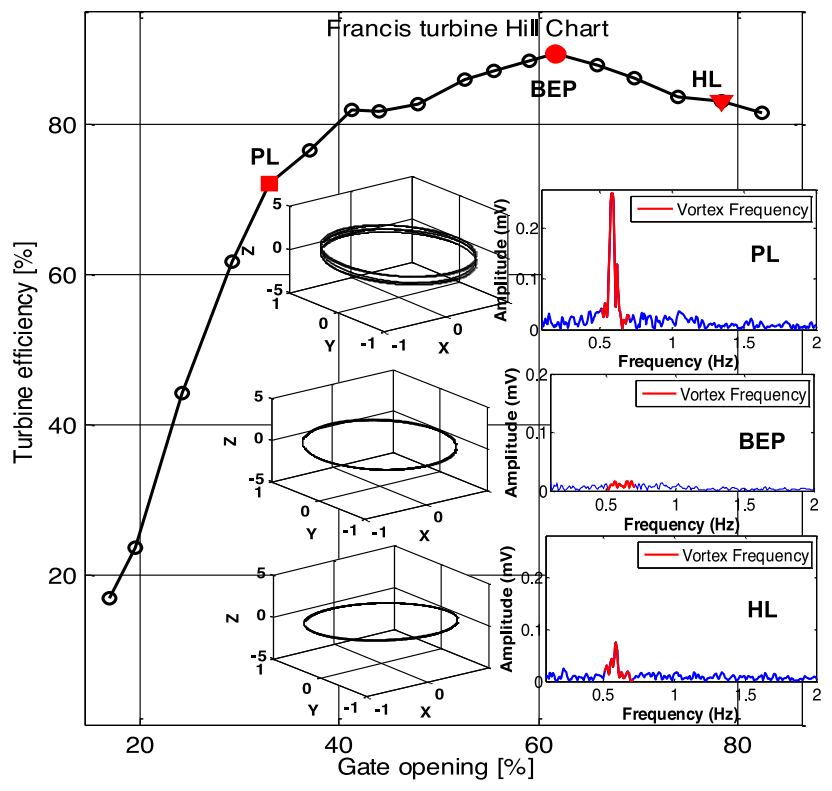

Fig. 7. Three-dimensional interpretation of the vortex cavitation appearance.

$\mathrm{X}$ is that the statistical moments $m_{1}$ and $m_{2}$ of $\mathrm{X}\left\{\mathrm{S}_{i n}\right\}$ are correlated and that they vary according to the cavitation vortex appearance.

One can notice a vortex detection event by the drop of the second order moment, $m_{2}$, for a gate opening between $25-50 \%$. The vortex presence and, hence appearance of its corresponding frequency, is marked by a lower data's second order moment. The first order moment do not provide enough information, especially that of $S_{i n}$. Using (3), the extensometer's vertical elongations are transposed to angular rotation of a circle with 3 degree of freedom: rotation around the $x, y$, and $z$ directions. In order to obtain a 3-D rotation of the circle, at every instance of time, a product of the three rotation matrices following (1) and (2), for each direction, is applied to a unitary circle in initial position. The rotation period is equal to the vortex rotation period. Equation (4) gives the spherical coordinates of the rotated circle, at each time instant $t$. In Fig. 7, we can visualize, on the turbine hill chart, the presence of the cavitation vortex. Three operating points are considered: part load (PL), the best efficiency point (BEP) and high load (HL). For PL, more than for HL, the vortex appearance is marked by the presence of low frequencies on signal's spectrogram, and by the deviation from the 3-D circle reference position given by the BEP.

In the case of cavitation vortex appearance, the statistical moments of the corresponding signal are close to those of a Gaussian pdf. This fact is in agreement with our initial assumption from Section II-A that the additive noise present in the cavitation vortex frequency bandwidth is considered to be Gaussian.

\section{CONCLUSION}

Cavitation vortex and all flow instabilities have a major impact on hydraulic machinery efficiency. The ability to detect instantly the flow parameters changes, improves the machines monitoring and thus, low efficiency exploitation can be avoided. In this paper, we have presented a new application for extensometer measurements, which provide real time information about the cavitation vortex appearance, using a mathematical model for data processing. Perspectives of study concern the adaptive estimation for the reference noise standard deviation [18].

\section{ACKNOWLEDGMENT}

The authors would like to thank J.-L. Ballester for his recommendations, as well as "Électricité de France" (EDF) for providing the experimental calibration data.

\section{REFERENCES}

[1] L. D. Landau and E. M. Lifschitz, Mechanics, New York, NY, USA: Pergamon, 1976, p. 113

[2] G. F. Round, Incompressible Flow Turbomachines: Design, Selection, Applications and Theory, Amsterdam. The Netherlands: Elsevier, 2004, pp. 55-102.

[3] J. P. Franc, Fundamentals of Cavitation, vol. 76. Haarlem, The Netherlands: Springer, 2005, p. 265.

[4] S. Houde, M. Iliescu, R. Fraser, S. Lemay, G. D. Ciocan, and C. Deschênes, "Experimental and numerical analysis of the cavitating part load vortex dynamics of low-head hydraulic turbines," in Proc. Fluids Eng. Div. Summer Meeting, ASME-JSME-KSME, Joint Fluids Eng. Conf., 2011, vol. 2, pp. 171-182. doi: 10.1115/AJK2011-33006.

[5] J. Paik, F. Sotiropoulos, and M. J. Sale, "Numerical simulation of swirling flow in complex hydroturbine draft tube using unsteady statistical turbulence models," J. Hydraul. Eng., vol. 131, no. 6, pp. 441-456, 2005.

[6] R. Susan-Resiga, G. D. Ciocan, I. Anton, and F. Avellan, "Analysis of the swirling flow downstream a Francis turbine runner," J. Fluids Eng., vol. 128, pp. 177-189 2006.

[7] S. Tridon, S. Barre, G. D. Ciocan, and L. Tomas, "Experimental analysis of the swirling flow in a Francis turbine draft tube: Focus on radial velocity component and possible applications determination," Eur. J. Mech., B/Fluids, vol. 25, no. 4, pp. 321-335, 2010.

[8] Z. Yuning, L. Kaihua, X. Haizhen, and D. Xiaoze, "A review of methods for vortex identification in hydroturbines," Renew. Sustain. Energy Rev., 2017. [Online]. Available: https://doi.org/10.1016/j.rser.2017.05.058

[9] X. Wang, M. Nishi, and H. Tsukamoto, "A simple model for predicting the draft tube surge," in Proc. 17th Int. Assoc. Hydro-Environ. Eng. Res. Symp., Beijing, China, Sep. 15-19, 1994, vol. 1, pp. 95-106.

[10] M. Iliescu, Analysis of Large Scale Phenomena in Turbine Draft Tubes, Ph.D dissertation, Lab. Hydraulic Machines, École Polytechn. Féd. Lausanne, Lausanne, Switzerland, 2007.

[11] D. N. Konidaris and J. A. Tegopoulos, "Investigation of oscillatory problems of hydraulic generating units equipped with Francis turbines," IEEE Trans. Energy Convers., vol. 12, no. 4, pp. 419-425, Dec. 1997.

[12] F. Hongqing, C. Long, D. Nkosinathi, and S. Zuyi, "Basic modeling and simulation tool for analysis of hydraulic transients in hydroelectric power plants," IEEE Trans. Energy Convers., vol. 23, no. 3, pp. 834-841, Sep. 2008.

[13] S. Denis, Analyse Dynamique D'une Ligne D'arbre Verticale Supportée Par Une Butée à Patins Oscillants, Ph.D. dissertation, Dept. Mech. Eng., Prod. Trans., Univ. Poitiers, Poitiers, France, 2014

[14] N. P. Cheremisinoff, Practical Statistics for Engineers and Scientists, Lancaster, PA, USA: Technomic, 1987, pp. 61-63.

[15] H. Ventsel, Théorie Des Probabilités, Moscow, Russia: Editions de Moscou, 1973, pp. 251-253.

[16] M. Abramowitz and I. Stegun, Handbook of Mathematical Functions with Formulas, Graphs and Mathematical Tables. New York, NY, USA: Dover, 1964, p. 302.

[17] M. Le Roux, G. Vasile, and T. Lora-Ronco, "Analyse de signaux issus de capteurs extensométriques de poussée hydraulique," Internship Rep., 2015. [Online]. Available: https://doi.org/10.13140/RG.2.2.26709.01768.

[18] D. Pastor and F. Socheleau, "Robust estimation of noise standard deviation in presence of signals with unknown distributions and occurrences," IEEE Trans. Signal Process., vol. 60, no. 4, pp. 1545-1555, Apr. 2012. 